\title{
OBSERVATIONS OF THE DIFFUSE ULTRAVIOLET BACKGROUND FROM THE UVX EXPERIMENT ON SPACE SHUTTLE
}

\author{
Richard C. Henry and Paul D. Feldman \\ Department of Physics and Astronomy \\ The Johns Hopkins University \\ Baltimore, MD 21218, USA
}

\author{
Jayant Murthy \\ Laboratory for Astronomy and \\ Solar Physics \\ Goddard Space Flight Center \\ MD 20771, USA
}

\begin{abstract}
The UVX experiment has resulted in confirmation that the diffuse ultraviolet background is low in intensity everywhere and that it is fairly uniform in intensity, both spatially and spectrally. There is no clear evidence that any significant portion of the moderate galactic latitude diffuse cosmic background originates in galactic plane starlight scattering from interstellar dust.
\end{abstract}

The first sensitive survey of the diffuse ultraviolet background over large portions of the sky, the Apollo 17 results of Anderson, Henry, and Fastie (1982), showed no trace of ultraviolet light scattered from dust, and they indicated that the cosmic background at all moderate and high galactic latitudes was -300 photon units or less. It thus came as quite a surprise when Paresce et al. (1979) and Paresce, Jakobsen, and Bowyer (1983) reported ultraviolet intensities, from the Berkeley Apollo-Soyuz experiment, of many thousands of photon units over large regions of the sky, and at all galactic latitudes. From among their 3200 observations, Paresce, McKee, and Bowyer (1980) located 128 that provided four different correlations of intensity with hydrogen column density, from which they concluded that scattering of galactic-plane starlight off dust grains is the primary source of the observed radiation field at intermediate latitudes. A more impressive case for a dust-scattering origin for the cosmic ultraviolet background is provided by Joubert et al. (1983).

This interesting situation (Berkeley reporting large diffuse-background intensities in some directions, and at least some correlation with HI column density, and Johns Hopkins reporting low intensities and no correlations) led to the UVX experiment, in which, on Space Shuttle flight STS-61C, co-aligned Johns Hopkins and Berkeley spectrometers observed the same nine celestial regions at the same time. The targets were chosen jointly.

Some of the UVX results have already been reported: Murthy, Henry, and Feldman (1989a) report an average brightness of only 375 photon units, with no target exceeding 700 photon units in brightness. These results are clearly consistent with the Berkeley "bright patches" being spurious, a conclusion that the earlier work of Weller (1983) had already strongly suggested.

What of correlations with HI? Murthy, Henry, and Feldman (1989b) find no correlation between ultraviolet intensity and HI column density. We now provide details (Figure 1) of a scan (target 6 of Murthy, Henry, and Feldman) from galactic latitude $26^{\circ}$ down to $9^{\circ}$. Sharp spikes are individual stars. (The spacecraft ceased its spatial scanning at $\sim 1900 \mathrm{~s}$; the rise observed after $2300 \mathrm{~s}$ must be geophysical, or dark-count-related). Our dark count was not measured in flight, and if it is as high for this target as Murthy, Henry, and Feldman (1989b) suggest, there is little or no signal that could be attributed to light scattering from dust. 


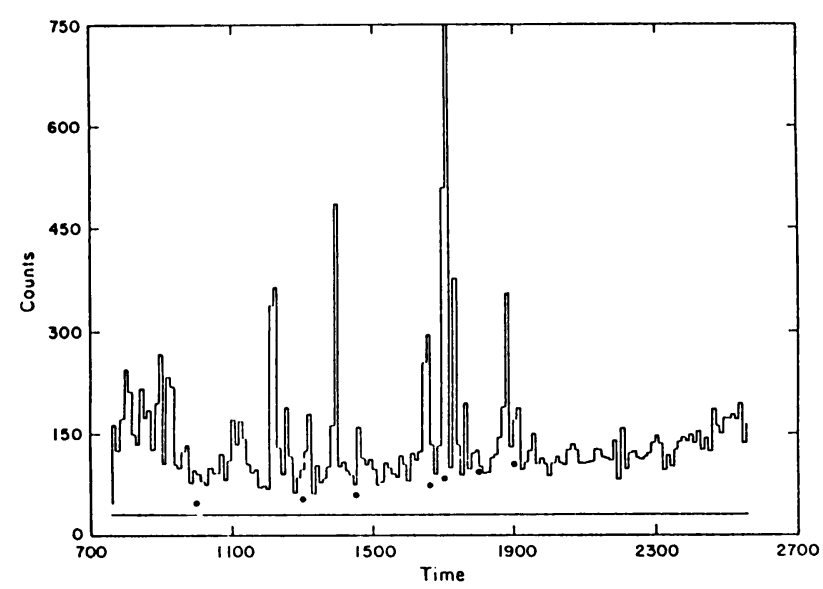

Figure 1. Intensity (1250-2500 $\AA$ ) versus time for The Johns Hopkins University spectrometer, for target 6 of the UVX mission.
In the present figure we explore the alternative hypothesis that the dark current was just as low for this target as it was for earlier UVX targets (horizontal line in figure), which also would give an average Johns Hopkins diffuse ultraviolet flux on this target very similar to the Berkeley flux for this target. The filled circles in Figure 1 are proportional to HI column density, on an arbitrary intensity scale. We assume dust density is proportional to HI. Saturation should set in at about $1500 \mathrm{~s}$; that is, little increase in diffuse UV intensity should occur after this time, despite the continuing increase in $21 \mathrm{~cm}$ column density.

Very little correlation appears in Figure 1 between the observed diffuse background and the $21 \mathrm{~cm}$, with or without attention to expected saturation. Thus, the Johns Hopkins UVX results continue to suggest that scattering from dust is only a minor contributor to the ultraviolet background at moderate galactic latitudes.

Acknowledgments-This work was supported by NASA grant NAG 5-619 to The Johns Hopkins University.

\section{REFERENCES}

Anderson, R. C., Henry, R. C., and Fastie, W. G. 1982, Ap. J., 259, 573.

Joubert, M., Masnou, J. L., Lequeux, J., Deharveng, J. M., and Cruvellier, P. 1983, Astr. Ap., 128, 114.

Murthy, J., Henry, R. C., Feldman, P. D., and Tennyson, P. D. 1989a, Ap. J., 336, 954.

Murthy, J., Henry, R. C., Feldman, P. D., and Tennyson, P. D. 1989b, Astr. Ap., (in press).

Paresce, F., Jakobsen, P., and Bowyer, S. 1983, Astr. Ap., 124, 300.

Paresce, F., Margon, B., Bowyer, S., and Lampton, M. 1979, Ap. J., 230, 304.

Paresce, F., McKee, C., and Bowyer, S. 1980, Ap. J., 240, 387.

Weller, C. S. 1983, Ap. J., $268,899$. 